Voix et Images

volxetimages

\title{
À quand une histoire de la pensée québécoise?
}

\section{Jacques Allard}

Volume 16, numéro 1 (46), automne 1990

Les correspondants littéraires d'Alfred DesRochers

URI : https://id.erudit.org/iderudit/200885ar

DOI : https://doi.org/10.7202/200885ar

Aller au sommaire du numéro

Éditeur(s)

Université du Québec à Montréal

ISSN

0318-9201 (imprimé)

1705-933X (numérique)

Découvrir la revue

Citer cet article

Allard, J. (1990). À quand une histoire de la pensée québécoise? Voix et Images, 16(1), 161-165. https://doi.org/10.7202/200885ar d'utilisation que vous pouvez consulter en ligne.

https://apropos.erudit.org/fr/usagers/politique-dutilisation/ 


\section{Recherches}

\section{À quand une histoire de la pensée québécoise?}

\section{par Jacques Allard, Université du Québec à Montréal}

Mon titre vient de cette impatience qui surgit à chaque fois qu'il me faut situer historiquement notre discours critique (littéraire, mais aussi philosophique, sociologique, etc). Quand l'aurons-nous enfin cette histoire de la pensée au Québec? N'en avez-vous pas assez d'être renvoyé à des centaines d'essais divers qu'il faudrait avoir tous lus, parce que personne n'en a encore fait la synthèse. Souvent on préfêre se comporter comme si on savait de quoi il retourne dans l'histoire de nos idées. Si vous ne l'avez encore tenté, faites-en l'expérience, rapide: consultez vos sources habituelles en critique ou en histoire littéraire, en recherchant les repères précis invoqués par les auteurs pour marquer les étapes historiques de notre pensée. Vous verrez que tout le monde fait comme si tout allait de soi dans une évolution socio-politique plus ou moins cléricalisée. Pour ma part, j'ai l'habitude de me rabattre sur certains ouvrages généraux, souvent non littéraires, pour savoir où me diriger dans les lectures croisées. Me guide souvent l'Histoire du Québec contemporain ${ }^{1}$ dont les auteurs ont bien intégré, me semble-t-il, les recherches "idéologiques" qui se font depuis trente ans, encore que les historiens d'aujourd'hui ignorent assez souvent le contenu des textes littéraires, les plus informés (c'est-à-dire: sous les angles habituels de la politique ou de la vie sociale et 'économique) étant parfois presque illettrés. C'est bien l'époque de l'art minimal, du clip et autres bips des spécialistes du peu. Je ne suis pas étonné (small is so beautiful, comme le dit depuis si longtemps notre slogan profond), seulement tanné: pour savoir penser, il vaudrait mieux savoir d'où l'on vient. 


\section{Une histoire des idées}

Tout ne va-t-il pas maintenant changer? Un collègue historien estimé, Yvan Lamonde, prépare peut-être l'ouvrage attendu, comme l'annonce la parution de l'Histoire des idées au Québec, 17601960: bibliographie des études ${ }^{2}$. Je donne le titre dans son intégrité, ce que ne fait pas, curieusement, l'éditeur (pourtant, c'est la Bibliothèque nationale) qui trompe le lecteur en'ne disant pas dès la couverture qu'il s'agit d'une bibliographie. Et encore faudrait-il préciser qu'il ne s'agit pas d'un travail simplement chronologique et signalétique; nous est plutôt proposée une bibliographie (selon le terme consacré dans les études littéraires: c'est-à-dire provenant d'une conceptualisation du domaine). Les huit cents références sont effectivement découpées en fonction de six périodes, qui chapeautent, ellesmêmes, de cinq à dix-huit rubriques, issues des champs ou thèmes relevés par l'historien et auteur.

On connaît les nombreuses contributions d'Yvan Lamonde dans le champ de l'histoire (même littéraire: sur les bibliothèques, par exemple). On se souviendra aussi de son ouvrage consacré à l'histoire de l'enseignement de la philosophie au Québec ${ }^{3}$ qui témoignait bien de son intérêt pour la vie des idées ici. C'est donc avec empressement qu'on entre dans son ouvrage illustré d'une quinzaine de documents et divisé en quatre parties (Table des matières, "Introduction", "Historiographie des mouvements intellectuels (1855-1985)", la bibliographie principale et l'" Index des auteurs").

Ce qui s'offre d'abord à la lecture, c'est la table des matières. Attention, c'est peut-être, déjà, l'essentiel. À la parcourir illico, on se rend compte de l'importance de son contenu. Cette table n'est pas que l'énumération fonctionnelle des parties d'un ouvrage. Là se livre le résultat d'un premier travail: la classification thématique et chronologique des études, le principe de la systématique en jeu ici. Cela se voit dans le choix de la périodisation et de ses sous-titres qui découpent les six sections du thesaurus. Lisons bien, car ce cadre fondamental de références n'est pas là présenté autrement que dans son énumération. En fait, c'est dans deux autres publications que l'on doit aller chercher l'éclairage nécessaire, la justification du plan de cette table. D'abord dans le bulletin de la Bibliothèque nationale, $\grave{A}$ rayons ouverts où Yvan Lamonde fait l'"Histoire d'une histoire " 4 (racontant son projet longuement mûri d'une histoire des idées au Québec), puis dans "L'histoire culturelle et intellectuelle du Québec: tendances et aspects méthodologiques " ${ }^{5}$ publié par le même auteur.

\section{D'une révolution à l'autre}

Je reprends donc l'énumération des titres de sections de la bibliographie, en comprenant que 1760-1815 = "Conquête et révolutions"; 1815-1840 = "L'essor du nationalisme et du libéralisme »; 1840-1880 = 
"Une vie intellectuelle active: l'antagonisme libéral-ultramontain"; 1880-1929 = "Les idées, la ville et l'usine"; 1929-1945 = "Une crise intellectuelle»; 1945-1960 = "Pour une pensée libre».

"Conquête et révolutions ». Peut-on discuter ce point de départ? ce mot de "conquête" à nouveau utilisé ici, comme chez tant d'autres historiens, m'étonnera toujours: n'est-ce pas voir le changement de régime par les yeux des conquérants? Pour ma part, je préférerais qu'on dise franchement: la Défaite, puisque c'est ce que nous avons vécu. J'essaie en vain d'imaginer les Français parler de la Conquête pour l'armistice de 1940 et la collaboration qui a suivi! $\grave{A}$ moins que ce tic de nos historiens veuille dire que nous avons participé à cette conquête de nous-mêmes? (Cf. la théorie aquinienne du Québécois comme agent double ou plus tard le Canadien français et son double - de Jean Bouthillette; 1972 - qui accréditent justement notre " collaboration" depuis deux cents ans...) J'hésite à m'engager dans la sémio- ou sémantico-psychanalyse que pratiquerait ici Heinz Weinmann ${ }^{6}$. De toute façon, l'utilisation du mot «Défaite " dirait plus clairement l'aplatissement démographique, social et culturel qui a conduit à notre recommencement collectif, sur la base d'une civilisation française rurale encore analphabète. En fait, le seul terme de "révolutions", qui est finalement le plus approprié, suffirait ici, voulant dire au sens premier qu'on oublie souvent quand on n'est pas astronome: mouvement par lequel on revient au point de départ. N'est-ce pas le premier sens des événements de 1760 ? C'est ce recommencement que nous allons bâtir avec, et surtout contre l'apport des révolutions états-unienne et française, telles que filtrées par un nouveau pouvoir colonial (anglo-protestant) et la domination cléricale catholique qui le relaiera. Autre remarque: comment 1815 serait-elle une date décisive pour l'histoire de notre pensée? La levée du blocus napoléonien est-elle plus importante que la guerre anglo-américaine (1812) ou que telle idée, contenue dans la Phénoménologie de l'esprit (Hegel; 1807), ou encore que la parution du journal le Canadien (en 1806)? Je m'étonnerai toujours (naïveté?) qu'il faille s'en remettre toujours à une datation socio-politique pour comprendre le mouvement des idées: elles s'éprouvent généralement dans une circulation plus restreinte, souvent confinée à des cercles (généralement intellectuels ou artistiques) avant de se cristalliser dans un État, une mode ou un programme politique. Et ces idées ne naissent pas forcément en rapport avec une événementialité strictement socio-politique. Aussi, ce que font trop souvent les historiens (est-ce depuis qu'ils se sont exclus du discours "littéraire» pour proposer un discours "scientifique"?), c'est de construire leur récit historique à partir des marques extérieures, socio-politiques, de la reconnaissance du mouvement intellectuel. Alors de quelle histoire s'agit-il finalement? Celle de la fortune socio-politique de telle pensée, ou telle idée? Il y a, là encore, matière à débat. 
Pour 1815-1840, je dirais tout aussi bien «naissance d'une conscience historique", pour bien marquer l'impact de la nouvelle idée propre au XIX ${ }^{e}$ siècle, ici comme ailleurs, celle d'un homme «historique", c'est-à-dire déployé dans le temps plutôt que dans l'irréel ou l'utopie. Et je mettrais le libéralisme en rapport avec cette nouvelle conscience. Pour l'étape 1840-1880, je dirais la rétrogradation amenée par l'utopisme ou l'idéalisme romantico-catholique au temps du réalisme. Et je parlerais ensuite de la longue bataille moderniste qui commence au tournant du siècle pour nous amener jusqu'à l'autre révolution, celle-là au sens second, de 1960. Je donnerais à chacune des rubriques une appellation idéelle ou philosophique plutôt que ce registre factuel qui s'accuse à partir de 1880-1929 ("Les idées, la ville et l'usine»). "D'une révolution à l'autre» serait ma systématique.

\section{Et la pensée de la fiction?}

Serais-je ainsi plus idéologisant qu'historien, plus que les spécialistes du domaine? Le système lamondien est évidemment ici un reflet des études déjà menées par l'ensemble de la communauté historienne. Glissons: quand on regarde l'abondante moisson recueillie par l'auteur, on est impressionné. Il n'a apparemment pas oublié beaucoup d'études. C'est évidemment du côté littéraire que les carences seront pour nous plus évidentes: une quarantaine de titres (sur les huit cents) qui ne prennent que rarement en compte la pensée de la fiction, disons celle de la littérature d'idées (comme le faisait encore feu Jean-Charles Falardeau). Et Dieu sait si notre premier siècle littéraire fut à idées, semblable en cela à la littérature française. Des exemples de la pensée de la fiction? Tardivel l'ultramontain fait, malgré lui, la promotion de plusieurs valeurs modernes dans Pour la patrie. Le chanoine Groulx ne respecte pas toujours le dogme catholique dans l'Appel de la race.

En ai-je assez dit pour attirer votre attention sur un nouvel instrument de travail susceptible de rendre de très grands services à la réflexion sur le discours québécois? Quand vous irez voir de près cette bibliographie, vous verrez aussi que son auteur repère, pour chaque époque, les conditions de la vie intellectuelle, en général et en régions: l'instruction, l'éducation, la langue, l'alphabétisation, l'imprimé, les bibliothèques, la librairie, les voyages, les associations, etc. Il passe en revue les débats et leurs lieux ou médias. Vous comprendrez à ce moment que son approche est globale et systémique. Comme il le dit dans l'article méthodologique déjà cité, son circuit est celui de la communication: production, diffusion, consommation des idées, voilà ce qui l'intéresse. Vous verrez aussi (dans cet article) qu'il reconnait l'importance de la vie des formes esthétiques. Vous conclurez donc que sa bibliographie reflète d'abord l'état de la recherche, pas forcément le plan de l'ouvrage qui s'écrit. Mais vous regretterez 
tout de même de devoir pásser par trois publications différentes pour prendre la mesure d'un projet fascinant, tout en vous demandant si cette histoire (sociale) des idées donnera pour autant une histoire de la pensée.

Qu'est-ce d'abord que la pensée, oserez-vous encore dire? Une activité de groupe? des idéologies? des philosophies? des visions du monde, d'individus ou de classes (dominantes et opposées)? celle qu'on trouve dans des ouvrages non seulement de philosophie, mais aussi de critique, de fiction et d'histoire (vraie!)? Sans doute tout cela et davantage, vous répondra la doxa scientifique, en recouvrant le tout (pour combien de temps?) d'un label réducteur, celui du discours social. Vous soutiendrez donc tout collègue qui se lance dans la première histoire de la pensée québécoise.

1 Paul-André Linteau, René Durocher, Jean-Claude Robert, Histoire du Québec contemporain, Montréal, Éditions du Boréal, tome I, De la confédération à la crise, 1979, 658 p. et tome II, le Québec depuis 1930, nouvelle édition révisée, Boréal compact, 1989, 834 p. (François Ricard s'ajoute aux trois auteurs déjà indiques). "Orientations bibliographiques " données à la fin des chapitres.

2 Yvan Lamonde, l'Histoire des idées au Québec, 1760-1960: bibliographie des études, Montréal, Bibliothèque nationale du Québec, 1989, $167 \mathrm{p}$.

3 La philosophie et son enseignement au Québec (1665-1920), Montréal, Hurtubise-HMH, 1980, 312 p. (Cahiers du Québec, $n^{\circ} 58$ ).

4 Yvan Lamonde, "Histoire d'une histoire", $\hat{A}$ rayons ouverts, bulletin de la Bibliothèque nationale, $2^{e}$ année, $n^{\circ} 7$, été 1989. (N.B.: distribué gratuitement, ce bulletin est très commode pour les chercheurs; on l'obtient en en faisant la demande à la Bibliothèque nationale, secteur des publications, 1700, rue SaintDenis, Montréal, Québec, H2X 3K6.)

5 Yvan Lamonde, "L'histoire culturelle et intellectuelle du Québec: tendances et aspects méthodologiques", Revue d'études canadiennes/Journal of Canadian Studies, vol XXIV, $\mathrm{n}^{\circ} 3$, automne 1989. Intitulé "L'histoire culturelle du Canada", ce numéro, largement dominé par des contributions anglophones sur le Canada anglais, contient, outre l'article cité plus haut, une intéressante revue des livres parus sur le Canada français (surtout en anglais, le seul francophone étant Textes de l'exode de Maurice Poteet) par Yves Frenette, lequel remarque une nette tendance à la hausse de l'intérêt des anglo-américains pour les "Québec Studies" (Meech aidant). On trouve aussi, en anglais, une critique très incisive de la tendance postmoderne de la recherche (sur la "gynocritique", l'art, la poétique, etc.) au Canada par Gail McGregor: "The Mainstreaming of Postmodernism: A Status Report on the "New" Scholarship in Canada".

6 Voir Heinz Weinmann, Du Canada au Québec, généalogie d'une histoire, Montreal, l'Hexagone, 1987 (ouvrage étonnant, passionnant, provocant); et Cinéma de l'imaginaire québécois. De la Petite Aurore à Jésus de Montréal qui vient de paraitre chez le même éditeur (et dont je discuterais davantage les analyses proposées). 